\title{
Age-Related Differences in Neuropsychological Assessment of Fetal Alcohol Spectrum Disorder: A Cross-sectional Study
}

\begin{tabular}{|r|l|}
\hline Journal: & Biochemistry and Cell Biology \\
\hline Manuscript ID & bcb-2017-0081.R1 \\
\hline Manuscript Type: & Article \\
\hline Complete List of Authors: & $\begin{array}{l}\text { Taylor, Nicole; University of Manitoba, Clinical Health Psychology; } \\
\text { Specialized Services for Children and Youth Centre, Manitoba FASD Centre } \\
\text { Enns, Leah; University of Manitoba, Clinical Health Psychology; Specialized } \\
\text { Services for Children and Youth Centre, Manitoba FASD Centre }\end{array}$ \\
\hline $\begin{array}{r}\text { Is the invited manuscript for } \\
\text { consideration in a Special } \\
\text { Issue? : }\end{array}$ & Fetal Alcohol Spectrum Disorder \\
\hline Keyword: & $\begin{array}{l}\text { fetal alcohol spectrum disorder, prenatal alcohol exposure, } \\
\text { neuropsychological assessment, age-related differences, childhood and } \\
\text { adolescence }\end{array}$ \\
\hline
\end{tabular}




\title{
Age-Related Differences in Neuropsychological Assessment of Fetal Alcohol Spectrum
} Disorder: A Cross-sectional Study

\author{
Nicole M. Taylor ${ }^{1,2}$ and Leah N. Enns ${ }^{1,2}$ \\ ${ }^{1}$ Department of Clinical Health Psychology, University of Manitoba, 771 Bannatyne Avenue, \\ Winnipeg, MB, R3E 3N4 \\ ${ }^{2}$ Manitoba FASD Centre, SSCY Centre, 1155 Notre Dame Avenue, Winnipeg, MB, R3E 3G1
}

Address correspondence to Leah Enns, Manitoba FASD Centre, SSCY Centre, 115 Notre Dame Avenue, Winnipeg, MB, R3E 3G1, e-mail: lenns@rccinc.ca 
Age-Related Differences 2

\begin{abstract}
This cross-sectional study examined six key areas of neuropsychological functioning (cognitive, academic, attention, executive function, adaptive skills) comparing adolescents and school-age children with prenatal alcohol exposure (PAE). The aims were: (1) to examine which neuropsychological measures were predictive of an FASD diagnosis in adolescents and schoolage children with PAE, and (2) to compare the neuropsychological performance of adolescents and children diagnosed with FASD. Hierarchical logistic regressions determined that the FullScale IQ, Verbal Comprehension and Perceptual Reasoning indices, basic reading and math skills, adaptive functioning at school, and components of executive functioning (dependent on age) improved the probability of an accurate FASD diagnosis in both groups: $9.1 \%$ to $19.2 \%$ for adolescence and $10.9 \%$ to $19.4 \%$ for school-age (61.5\%-80.9\% correct classifications overall). For the age comparison analyses (ANOVAs/MANOVAs), a significant difference was observed in the cognitive domain, as well as with basic math skills (trend) in the FASD diagnosed sample, with lower scores observed for adolescents across these measures. These findings provide further evidence for age differences in neuropsychological assessment as well as increased neuropsychological difficulties in adolescence compared to childhood with FASD. Longitudinal studies will be needed in order to make further inferences about developmental changes in neuropsychological functioning in FASD.
\end{abstract}

Key Words: fetal alcohol spectrum disorder, prenatal alcohol exposure, neuropsychological assessment, age-related differences, childhood and adolescence 
Age-Related Differences 3

\section{Age-Related Differences in Neuropsychological Assessment of FASD: A Cross-sectional}

\section{Study}

Fetal Alcohol Spectrum Disorder (FASD) includes a wide range of neurobehavioural and developmental abnormalities and is estimated to affect between 1 and 3\% of Canadians (Health Canada, 2006; Roozen et al. 2016). Individuals with FASD demonstrate significant impairments across a number of CNS functions (e.g., general intelligence, language and communication, memory, executive functioning, level of adaptive functioning, motor functioning, etc.; Chudley, Conry, Cook, Loock, Rosales, \& LeBlanc, 2005; Cook et al. 2016). As children with FASD enter adolescence, impairments in these different areas of functioning can lead to a myriad of challenging outcomes, including higher rates of mental health problems, higher likelihood of school dropout, increased risk-taking and impulsive behaviours, and problems with the law (e.g., Hanlon-Dearman, Green, Andrew, LeBlanc, \& Cook, 2015; Hellemans, Sliwowska, Verma, \& Weinberg, 2010; Streissguth, Bookstein, Barr, Sampson, O’Malley, \& Young, 2004). It is not clear whether the increase in functional impairments that are observed in adolescence stem from secondary outcomes that emerge from increased demands typically present during this developmental period (e.g., increasing autonomy, responsibility, requirement for planning and self-management), or whether alterations in normal brain developmental processes are at play. Are adolescents with FASD failing to meet the increased expectations associated with 'growing up,' or are they failing to develop at the same rate or in the same manner as their non-affected peers?

Strikingly little is known regarding the neural and neuropsychological trajectories of individuals with FASD through childhood, into adolescence, and beyond. Perhaps the strongest evidence for persisting effects of prenatal alcohol exposure (PAE) with increased age comes 
from the work of Streissguth and colleagues (see Streissguth, 2007 for review), who used a prospective longitudinal paradigm to follow a cohort of approximately 500 prenatally-alcohol exposed children from birth to 25 years. In childhood, reductions in overall intelligence and speed of information processing as well as difficulties with attention, arithmetic, and visualspatial memory were associated with PAE. In adolescence, aspects of executive functioning skills (e.g., decision making, following directions, and task persistence) were associated with PAE. Impairments persisted over time and a number of deficits identified early on, particularly those related to attention, vigilance and motor skills, were related to deficits apparent later. This work was paramount to understanding dose-dependent and (likely) lifelong impacts of PAE. Follow-up studies from Helsinki (Autti-Ramö, 2000) and Berlin (see Steinhausen \& Spohr, 1998) corroborated the persistence of psychopathology in FASD over development, including social-adaptive problems, cognitive impairment, and attention-related disturbance. In the 20 year follow-up Berlin FAS Study, preschoolers showed a preponderance of attention-related disturbance, enuresis, and eating disorders, while in childhood there was an emergence of language disorders, sleep disorders, abnormal behaviours, and emotional disorders. A reduction of language disorders and an increase in emotional disturbances characterized adolescents with FASD. Attention-related disorders and social relationship problems were persistently high over development. For the most part, intellectual functioning remained stable over time; however, for a sizeable proportion of children, intelligence quotients decreased with age. Given the emphasis on tracking deficits at specified timepoints rather than charting developmental trajectories per se, this series of work did not provide detailed evidence of age-related changes in FASD.

More recently, a number of studies have demonstrated a pattern of greater neuropsychological impairment for adolescents with FASD than for children with FASD (e.g., 
Rasmussen \& Bisanz, 2009; Tamana et al. 2014). These included performance on the DelisKaplan Executive Function System (DKEFS; Delis, Kaplan, \& Kramer, 2001) letter fluency, inhibition/switching, and word context tasks. Tamana and colleagues also identified poorer performance (compared to the norm) among the older group on arithmetic, verbal learning, visual-motor integration, motor tests, and Trail Making tests. The authors acknowledged that while the cross-sectional design of their study precluded drawing inferences surrounding changes in neuropsychological functioning with age, the findings do suggest that adolescents with FASD perform relatively poorer than children with FASD on a number of neuropsychological measures. Knowing more about the neuropsychological variables that are most likely to be affected in adolescence helps improve our understanding about the specific needs of adolescents with FASD. This, in turn, informs interventions that would be most suitable during this developmental period. Thus, the aims of the present study included: (1) describing the cognitive, academic, attention, executive functioning, and adaptive functioning profiles of adolescents with PAE assessed for FASD separately from school-age children also assessed for FASD, (2) identifying which measures most accurately differentiate a diagnostic outcome between adolescents and children with PAE, and (3) comparing neuropsychological performance across age groups among those diagnosed with FASD.

\section{Method}

All participants were referred from caregivers, schools, physicians, or child and family services to the Manitoba FASD Centre in Manitoba, Canada. All children seen at the Centre have a confirmed history of PAE as outlined in the 2005 Canadian guidelines (Chudley et al. 2005) and had significant issues with behaviour, development, and/or school performance. Further details about the diagnostic process can be found in Enns and Taylor (2016). A sample of 238 
children and adolescents with PAE (6.00-17.92 years; Mean age $=10.22(3.15) ; 60.5 \%$ boys) assessed between 2010 and 2015 were included in the present study. 55.9\% received an FASD diagnosis. To examine potential age-related differences in the predictiveness of measures used in the neuropsychological assessment of FASD, the sample was divided into school-age and adolescent diagnosed (PAE-FASD) and non-diagnosed (PAE) groups. Descriptive data for the socio-demographic information and assessment measures are included in Table 1. Chi-square tests and an ANOVA were completed to ensure the groups were comparable on several sociodemographic variables, including sex, caregiver at time of diagnosis (e.g., birth family, foster family, adoptive family, extended family), other diagnoses (e.g., ADHD, intellectual disability, learning disability, anxiety), and experience of psychosocial stressors and adverse life events (e.g., abuse, neglect, abandonment, family violence, significant losses, multiple caregivers). Chisquare analyses showed no differences between samples for sex, caregiver, or other diagnoses $\left(\chi^{2}\right.$ $=0.18-2.65, p=.10-.95)$. An ANOVA showed that psychosocial stressors were significantly related to age, $F(3,230)=3.26, p<.05$. Scheffe post-hoc comparison tests indicated a trend in that undiagnosed school-age children tended to experience more psychosocial stressors than diagnosed children $(p=.07)$, but that psychosocial stressors did not differ among adolescent groups.

The present study included a sampling of measures used to assess the cognitive, academic achievement, executive functioning, attention, and adaptive functioning domains examined as part of an FASD diagnostic assessment with criteria based on the 2005 Canadian diagnostic guidelines (Chudley et al. 2005). Canadian norms were used when available. The Full-Scale IQ, as well as the Verbal Comprehension, Perceptual Reasoning, Working Memory, and Processing Speed Indices from the Wechsler Intelligence Scale for Children, $4^{\text {th }}$ Edition (WISC-IV; 
Wechsler, 2003) or the Wechsler Adult Intelligence Scale, $4^{\text {th }}$ Edition (WAIS-IV; Wechsler, 2008) were included to evaluate the cognitive domain. Academic achievement was evaluated using the Word Reading, Pseudoword Decoding, Reading Comprehension, and Math Problem Solving subtests from the Wechsler Individual Achievement Test, $2^{\text {nd }}$ or $3^{\text {rd }}$ Editions (WIAT-II/III; Wechsler, 2005; Wechsler, 2009), or the Math Calculations, Word Reading, and Spelling subtests from the Wide Range Achievement Test 4 (WRAT4; Wilkinson \& Robertson, 2006). To maximize sample size, the Numerical Operations subtest from the WIAT-II/III and the Math Calculations subtest from the WRAT4 were combined into a "Math Computations" variable, as were the Spelling subtests from the WIAT-II/III and WRAT4. The School Problems scale from the Behavior Assessment Scale for Children, $2^{\text {nd }}$ Edition (BASC-2; Reynolds \& Kamphaus, 2004) teacher form was also examined. The attention domain was represented by the Conners-3 Inattention and Hyperactivity/ Impulsivity parent and teacher rating scales (Conners, 2008). The executive functioning domain was examined using scores from the DKEFS (Delis et al. 2001), including the Trail Making (Conditions 1 through 4, Visual Scanning, Number Sequencing, Letter Sequencing, and Letter-Number Sequencing, Condition 4 Total Error Rate), Verbal Fluency (Letter Fluency, Category Switching Correct Responses, Category Switching Total Accuracy Score), and Tower (Total Achievement Score) tests. In addition, the Global Executive Composites from the Behavior Rating Inventory of Executive Function (BRIEF; Gioia, Isquith, Guy, \& Kenworthy, 2000) parent and teacher rating scales were included in the present set of analyses. Finally, the adaptive functioning domain was represented by the Adaptive Skills Indices from the BASC-2 parent and teacher rating scales.

Although all children and adolescents were administered a number of the same standardized tests, not all completed every test due to age constraints, their level of functioning, 
Age-Related Differences 8

having completed previous psychology assessments, time, or testing fatigue. Therefore, only subsets of the total number of children were analyzed depending on the number of tests that were completed (see Table 1).

\section{Results}

All data were analyzed using IBM ${ }^{\circledR}$ SPSS $®$ Statistics 24 for Windows and continuous variables were standardized to a common metric. Bivariate correlations were initially completed for each age group separately to identify which neuropsychological assessment variables were significantly related to an FASD diagnosis. Variables that were significantly related to diagnoses for both groups included the Full-Scale IQ, Verbal Comprehension Index, Perceptual Reasoning Index, Reading Comprehension, Word Reading, Math Problem Solving, Math Computations, and BASC-2 Adaptive Skills teacher rating scale $(r=-.48$ to $-.27, p=.00-.05)$. Variables uniquely related to the school-age group included the Spelling test, BASC-2 School Problems Index teacher rating scale, and several measures from the DKEFS (Trail Making Condition 2 Number Sequencing, Letter Fluency Total Correct, Category Switching Total Correct Responses, and Category Switching Total Switching Accuracy; $r=-.43$ to $.25, p<.01$ ). Variables uniquely related to the adolescent group included two DKEFS measures, Trail Making Condition 1 Visual Scanning and Condition 3 Letter Sequencing $(r=-.40$ and $-.41, p<.01)$. All variables were assessed for multi-collinearity of $r>.70$. When found, the variables were examined for their commonality to both groups and their sample size. Those that were common to both groups or that had a larger sample size were chosen for subsequent analyses. For the final part of this study, mean comparisons were conducted on the subsample of FASD diagnosed school-age and adolescent groups. 
Following preliminary analyses, hierarchical logistic regressions were conducted, controlling for number of psychosocial stressors. To reduce the number of analyses, variables common to their respective domains were included in the final step (see Table 2 for a summary of regressions and variables included in each analysis $)$. All final models were significant $\left(\chi^{2}=\right.$ 5.98-38.60, $p=.00-.05)$. Results showed that the Full-Scale IQ, Verbal Reasoning and Perceptual Reasoning Indices, Word Reading and Math Computations, and Adaptive Skills teacher rating scales improved diagnoses between $10.9 \%$ and $15.4 \%$ for the school-age group, and between $9.1 \%$ and $14.3 \%$ for the adolescent group. When examining the individual variables across groups, the Verbal Comprehension Index was significantly related to an FASD diagnosis, while the Perceptual Reasoning Index was not. Furthermore, the Word Reading subtest, when entered in the same step as the Math Computations variable, was not significant across groups. The Math Computations variable was significantly related to diagnosis in the school-age group, but only tended toward significance for the adolescent group. The number of psychosocial stressors was generally significantly related to diagnosis for the school-age group and tended to be non-significant for the adolescent group.

Logistic regressions were also completed for the variables uniquely related to each age group. All final models were significant $\left(\chi^{2}=14.83-27.65, p=.00\right)$. For the school-age group, the four executive functioning variables from the DKEFS that were significantly correlated to an FASD diagnosis were entered in the same step (Trail Making Condition 2, Letter Fluency Total Correct, Category Switching Total Correct Responses, Category Switching Total Switching Accuracy). This model improved diagnostic accuracy by $19.4 \%$. When examined individually, the Category Switching Total Correct Responses score was significantly related to a diagnosis, while the Trail Making subtest tended toward significance. The regression including the School 
Problems Index improved diagnostic accuracy by $11.0 \%$. An additional logistic regression was also completed for the adolescent group, examining executive functioning as measured by the Trail Making subtests, Conditions 1 and 3. This model improved diagnostic accuracy by 19.2\%; however, individual subtests only tended toward significance.

For the final part of this study, age comparison analyses within the FASD school-age children and adolescent diagnostic groups were conducted. For these analyses, the common variables across age groups were examined using ANOVAs and MANOVAs as appropriate (Full-Scale IQ, Adaptive Skills teacher rating scale, Verbal Comprehension and Perceptual Reasoning Indices, Word Reading and Math Computations). Please see Figure 1 for graphical representation of these findings. For each graph, the $\mathrm{x}$-axis is the age group and the $\mathrm{y}$-axis is the standard scores. A significant difference was observed in the Full-Scale IQ, $\mathrm{F}(1,123)=5.28, p<$ $.05 ; \eta_{\mathrm{p}}{ }^{2}=.041$, but not the Adaptive Skills teacher rating scale, $\mathrm{F}(1,69)=0.97, p>.10 ; \eta_{\mathrm{p}}{ }^{2}=$ .014. Significant differences were also observed in the Verbal Comprehension Index, $F(1,125)=$ $12.71, p<.001 ; \eta_{\mathrm{p}}{ }^{2}=.092$, and Perceptual Reasoning Index, $\mathrm{F}(1,125)=4.81, p<.05 ; \eta_{\mathrm{p}}{ }^{2}=$ .037. When examining the common academic achievement subtests, findings were nonsignificant for the Word Reading subtest, $\mathrm{F}(1,114)=0.73, p>.10 ; \eta_{\mathrm{p}}{ }^{2}=.006$, and tended toward significance for the Math Computations variable, $\mathrm{F}(1,114)=3.23, p=.075 ; \eta_{\mathrm{p}}{ }^{2}=.028$. Across analyses, adolescents with an FASD diagnosis had lower scores than school-age children with FASD.

\section{Discussion}

The present study identified a number of neuropsychological variables that were related to an FASD diagnosis across the cognitive, academic, executive functioning, and adaptive functioning domains in both school-age and adolescent groups. These findings are consistent 
with previous research that has demonstrated broad differences in neuropsychological functioning between those with and without an FASD diagnosis (e.g., Enns \& Taylor, 2016; Nash et al. 2013; Rasmussen \& Bisanz, 2009).

\section{Neuropsychological Predictors of FASD in Childhood and Adolescence}

Across age groups, we identified a subset of variables that commonly predicted an FASD diagnosis, including overall IQ, verbal and nonverbal reasoning skills, basic reading and math skills, and adaptive skills in the school setting. Furthermore, there was a similar improvement in diagnostic accuracy in both groups, with the school-age group ranging from $10.9 \%$ to $15.4 \%$ and the adolescent group ranging from $9.1 \%$ to $14.3 \%$.

While the executive functioning domain was also common to an FASD diagnosis across groups, the measures that were actually predictive in each age group differed. Executive functions comprise those skills that support goal-directed behaviour. In the adolescent group, visual scanning and letter sequencing combined in a predictive model improved diagnostic accuracy by $19.2 \%$. While the school-age group's measures of executive function had a similar rate of diagnostic improvement (19.4\%), the executive functions were more related to number sequencing and verbal fluency, including lexical and category-switching skills. Across both age groups, individuals with poorer executive functioning skills were more likely to receive a diagnosis of FASD. As in Mattson et al. (2010, 2013) these findings suggest that executive functioning measures differentiate diagnosed from non-diagnosed individuals, but also suggest that there may be age-related differences in the measures that do so. This is consistent with other studies citing a differential association between executive functioning skills and PAE (Enns \& Taylor, 2016; Khoury, Milligan, \& Girard, 2015; Rasmussen \& Bisanz, 2009). Specifically, Khoury et al. (2015), using a meta-analytic review, identified a variety of variables, including the 
specific psychometric measures utilized as well as the nature of the groups under comparison, that factor into the magnitude of effect sizes for executive functioning tasks in different PAE groups. These findings suggest that while formal executive functioning measures provide a useful contribution to diagnostic clarification, the specific indicators that are most valuable vary according to age group.

Of note, caregiver and teacher ratings of applied executive functioning skills and attention-related problems did not contribute to increased diagnostic accuracy. This finding should not imply that children diagnosed with FASD did not experience impaired attention regulation or executive dysregulation in their day-to-day lives, but rather, that these problems figure prominently among referrals to our clinic and are behaviours often cited in the PAE population. As such, the non-diagnosed PAE-only group would have similarly high levels of attention-related disturbance as the FASD group. Indeed, review of Table 1 shows that for parent and teacher ratings of global executive functioning skills were in the Clinically Significant range across groups. Similarly, parent ratings of attention-related problems were in the Clinically Significant range while teacher ratings were in the At-Risk to Clinically Significant range across groups.

The finding that teacher (but not parent) ratings of adaptive problems across both groups, as well as school problems for the school-age group, contributed to increased diagnostic accuracy is consistent with previous research (Enns \& Taylor, 2015; Stevens, Nash, Fantus, Nulman, Rovet, \& Koren, 2013). Adaptive skills reflect those skills that are required for ageappropriate self-care and maintenance of activities of daily living. These findings suggest that incorporating teacher ratings at an early stage of the FASD assessment process (e.g., during an intake or screening phase) may help to inform the diagnostic assessment plan and thus allow for 
better individualization of the assessment, as well as potentially streamlining the assessment wait time.

\section{Age Differences}

Mean comparison analyses (as shown in Figure 1) identified age differences in the FASD subsample, particularly within the cognitive domain. Analyses showed that the adolescent group diagnosed with FASD had lower overall IQ scores, as well as poorer verbal comprehension and perceptual reasoning scores. A trend also emerged with respect to basic math computation skills, in that the adolescent group tended to have lower math scores. Age-related differences were not apparent across tests of word reading or teacher ratings of adaptive skills.

These findings complement those of Tamana et al. (2014), showing age-related changes across a variety of complex neuropsychological tasks. As in our study, Tamana et al. found agebased differences in arithmetic but not in word reading ability. Evaluation of potential agerelated differences in executive functions in our study was hampered by insufficient sample sizes, but has emerged as relatively weaker for adolescents with FASD than children with FASD in other research (Rasmussen \& Bisanz, 2009; Tamana et al. 2014). Further, as described above, we identified a different set of executive functioning skills that played a predictive role towards FASD diagnosis in childhood than in adolescence for our sample. The fact that intelligence factors (overall IQ, perceptual reasoning, and verbal comprehension) also showed age-related changes is a novel finding and speaks to the need to chart developmental factors (neural underpinnings, neuropsychological functions, functional abilities) using a prospective longitudinal design.

In recent years, a number of longitudinal MRI studies have pointed to altered trajectories of brain maturation (e.g., reduced cortical plasticity, delayed or altered white matter 
development) from childhood through adolescence in individuals with heavy in utero alcohol exposure (e.g., Lebel et al. 2012; Treit, Lebel, Baugh, Rasmussen, Andrew, \& Beaulieu, 2013). In other studies, age-related alterations in grey and white matter development were not found; however, age-related alterations in patterns of brain activation or brain-behaviour relationships among those with FASD were nevertheless observed (Gautam et al. 2014, 2015). In the latter studies, children and adolescents with FASD show aberrations in cortical structure that persisted over time, but did not appear to worsen (or improve).

\section{Limitations and Future Directions}

There are several limitations to the present study. First, while the combined sample size is quite large $(\mathrm{n}=239)$, the number of children and adolescents assessed using each measure is much more variable and resulted in a significantly smaller subset of samples used in the analyses. Given that assessments were performed for clinical purposes and thus data was collected retrospectively, not all measures were collected for each child. Factors such as fatigue, time constraints, and different age ranges for certain measures (e.g., DKEFS) meant different reductions in sample size across measures that would allow for meaningful analyses. Furthermore, these findings are not exhaustive and conclusions to be drawn are limited to the particular psychometric tests that were utilized in these assessment batteries. Further study would be needed to understand the predictive utility of other tests, such as those that measure language or motor skills, and how their inclusion changes the relative predictions of other tests and overall models. Second, the number of clinic-based referrals is not consistent across the age span. This was particularly true for the adolescent group. While comparable to the school-age group on socio-demographic variables, a larger sample of adolescents is needed to provide the power required to undertake more detailed and comprehensive analyses of the predictiveness of 
neuropsychological assessment at this developmental stage, and to evaluate age-related changes across a larger set of measures. Thirdly, the cross-sectional design of this study limits conclusions to understanding differences between age groups rather than age-related changes per se.

Despite the limitations, the present study contributes to the literature by using multiple methodologies, including mean comparisons and analyses that identify the predictiveness of neuropsychological measures used to diagnose FASD in PAE populations across development stages. The present study may have provided more specificity to where these differences lie, or more aptly, in which development stage certain skills and abilities are more predictive of an FASD diagnosis (i.e., more in an adolescent population than a school-age population).

Future research regarding the neuropsychological evaluation piece of the FASD diagnostic assessment may wish to consider differential weighting of assessment findings based on their predictive value, which may also prove helpful in the quest for efficient evaluations and accurate diagnosis. Further, as mentioned, little is known regarding developmental changes in FASD. This cross-sectional study, together with other findings, hints at the measures that could be evaluated using a longitudinal paradigm. Longitudinal MRI studies are underway to further understand brain-based changes across development in FASD. Future studies should consider the inclusion of more extensive neuropsychological batteries as well as an increased number of timepoints of assessment in order to capture the relative timing of potential developmental changes, which likely differ across different areas of functioning.

\section{Conclusions}

The goals of the present study were to identify the neuropsychological variables that were associated with and predictive of an FASD diagnosis among children and adolescents with 
FASD, and to identify any differences in performance on those variables between childhood and adolescence. First, given the in-depth assessment process, knowledge regarding the psychological measures that provide the most clarity around diagnosis is invaluable. This includes knowing which neuropsychological tools are most informative across the developmental spectrum. Specifying the most efficient testing batteries for accurately identifying a diagnosis of FASD in adolescence versus childhood may help guide assessment choices and the weight applied to different measures in the diagnostic clinics. It may also improve screening so we can better maximize the efficiency and efficacy of the assessment process and treatment recommendations. Second, by developing a better understanding of age-related differences in neuropsychological functioning, we can better address the needs of this complex population. Knowledge of these differences would then inform the development of age-appropriate treatments and accommodations, as well as improve the ability for professionals to provide anticipatory guidance, thus providing opportunity for early intervention and prevention. 


\section{Acknowledgements}

Both authors contributed equally to the development of this research project and manuscript. We appreciate the support the Manitoba FASD Centre and the University of Manitoba, Rady Faculty of Health Sciences, Max Rady College of Medicine, and Department of Clinical Health Psychology have provided to our lab. We also extend our gratitude to Kevin Solar, Pinar Eskicioglu, and Kristi MacDonald for their help with data entry. Finally, we are most indebted to the participants in the study.

There was no potential conflict of interest to report. 
Age-Related Differences 18

\section{References}

Autti-Ramö, I. (2000). Twelve-year follow-up of children exposed to alcohol in utero. Dev Med Child Neurol 42(6): 406-11.

Chudley, A.E., Conry, J., Cook. J.L., Loock, C., Rosales, T., and LeBlanc, N. (2005). Fetal alcohol spectrum disorder: Canadian guidelines for diagnosis. CMAJ 172: S1-S21. doi: 10.1503/cmaj.1040302

Conners, C.K. (2008). Conners 3 ( $3^{\text {rd }}$ ed.). Toronto, Ontario, Canada: Multi-Health Systems.

Cook, J.L., Green, C.R., Lilley, C.M., Anderson, S.M., Baldwin, M.E., Chudley, A.E., ... Rosales, T. (2016). Fetal alcohol spectrum disorder: A guideline for diagnosis across the lifespan. CMAJ 188(3): 191-197. doi: 10.1503/cmaj.141593

Delis, D.C., Kaplan, E., and Kramer, J.H. (2001). Delis Kaplan Executive Function System. San Antonio, TX: The Psychological Corporation.

Enns, L.N., and Taylor, N.M. (2015, March). Categorical differences between caregiver and teacher ratings of socio-emotional and adaptive functioning and subsequent FASD diagnoses. Paper session presented at the $6^{\text {th }}$ International Conference on Fetal Alcohol Spectrum Disorder, Vancouver, B.C., Canada.

Enns, L.N., and Taylor, N.M. (2016). Factors predictive of a fetal alcohol spectrum disorder: Neuropsychological assessment. Child Neuropsychol. 10: 1-23. doi: $10.1080 / 09297049.2016 .1251894$

Gautam, P., Nuñez, S.C., Narr, K.L., Kan, E.C., and Sowell, E.R. (2014). Effects of prenatal alcohol exposure on the development of white matter volume and change in executive function. Neuroimage Clin. 4(5): 19-27. doi: 10.1016/j.nicl.2014.05.010 
Gautam, P., Nuñez, S.C., Narr, K.L., Mattson, S.N., May, P.A., Adnams, C.M., ... Sowell, E.R. (2015). Developmental trajectories for visuo-spatial attention are altered by prenatal alcohol exposure: A longitudinal FMRI study. Cereb Cortex 25(12): 4761-4771. doi: $10.1093 /$ cercor/bhu 162

Gioia, G.A., Isquith, P.K., Guy, S.C., and Kenworthy, L. (2000). The Behavior Rating Inventory of Executive Function. Lutz, FL: Psychological Assessment Resources.

Hanlon-Dearman, A., Green, C.R., Andrew, G., LeBlanc, N., and Cook, J.L. (2015). Anticipatory guidance for children and adolescents with fetal alcohol spectrum disorder (FASD): Practice points for primary health care providers. J Popul Ther Clin Pharmacol 22(1): e27-e56.

Health Canada. (2006, September). It's your health: Fetal alcohol spectrum disorder, Minister of Health. Retrieved March 11, 2016 from http://www.hc-sc.gc.ca/hl-vs/iyh-vsv/diseasesmaladies/fasd-etcaf-eng.php

Hellemans, K.G.C., Sliwowska, J.H., Verma, P., and Weinberg, J. (2010). Prenatal alcohol exposure: Fetal programming and later life vulnerability to stress, depression and anxiety disorders. Neurosci. Biobehav. Rev. 34(6): 791-807. doi: 10.1016/j.neubiorev.2009.06.004

IBM Corp. Released 2016. IBM SPSS Statistics for Windows, Version 24.0. Armonk, NY: IBM Corp.

Khoury, J.E., Milligan, K., and Girard, T.A. (2015). Executive functioning in children and adolescents prenatally exposed to alcohol: A meta-analytic review. Neuropsychol. Rev. 25(2): 149-170. doi: 10.1007/s11065-015-9289-6

Lebel, C., Mattson, S.N., Riley, E.P., Jones, K.L., Adnams, C.M., May, P.A., ... Sowell, E.R. (2012). A longitudinal study of the long-term consequences of drinking during pregnancy: 
Heavy in utero alcohol exposure disrupts the normal processes of brain development. J.

Neurosci. 32(44): 15243-15251. doi: 10.1523/JNEUROSCI.1161-12.2012

Mattson, S.N., Roesch, S.C., Fagerlund, A., Autti-Rämö, I., Jones, K. L., May, P. A., ... Riley, E.P. (2010). Toward a neurobehavioral profile of fetal alcohol spectrum disorders. Alcohol Clin Exp Res 34(9): 1640-1650. doi: 10.1111/j.1530-0277.2010.01250.x

Mattson, S.N., Roesch, S.C., Glass, L., Deweese, B. N., Coles, C. D., Kable, J. A., ... Riley, E.P. (2013). Further development of a neurobehavioral profile of fetal alcohol spectrum disorders. Alcohol Clin Exp Res 37(3): 517-528. doi: 10.1111/j.1530-0277.2012.01952.x

Nash, K., Stevens, S., Rovet, J., Fantus, E., Nutman, I., ... and Koren, G. (2013). Toward identifying a characteristic neuropsychological profile for fetal alcohol spectrum disorders 1. Analysis of the Motherisk FASD Clinic. J Popul Ther Clin Pharmacol 20(1): e44-e52.

Ramsden, S., Richardson, F.M., Josse, G., Thomas, M.S., Ellis, C., Shakeshaft, C., ... Price, C.J. (2011). Verbal and non-verbal intelligence changes in the teenage brain. Nature 479(7371): 113-116. doi: 10.1038/nature10514

Rasmussen, C., and Bisanz, J. (2009). Executive functioning in children with fetal alcohol spectrum disorders: Profiles and age-related differences. Child Neuropsychol. 15(3): 201215. doi: $10.1080 / 09297040802385400$

Reynolds, C.R., and Kamphaus, R.W. (2004). Behavior Assessment System for Children (2 ${ }^{\text {nd }}$ ed.). Circle Pines, MN: American Guidance Service

Roozen, S., Peters, G-J.Y., Kok, G., Townend, D., Nijhuis, J., and Curfs, L. (2016). Worldwide prevalence of Fetal Alcohol Spectrum Disorders, A systematic literature review including Meta-Analysis. Alcohol Clin Exp Res 40(1): 18-32. doi: 10.1111/acer.12939 
Steinhausen, H.-C., and Spohr, H.-L. (1998). Long-term outcome of children with Fetal Alcohol Syndrome: Psychopathology, behavior, and intelligence. Alcohol Clin Exp Res 22(2): 334338. doi:10.1111/j.1530-0277.1998.tb03657.x

Stevens, S.A., Nash, K., Fantus, E., Nulman, I., Rovet, J., and Koren, G. (2013). Towards identifying a characteristic neuropsychological profile for fetal alcohol spectrum disorders: 2. Specific caregiver- and teacher-rating. J Popul Ther Clin Pharmacol 20(1): e53-e62. Streissguth, A.P., Bookstein, F.L., Barr, H.M., Sampson, P.D., O’Malley, K., and Young, J.K. (2004). Risk factors for adverse life outcomes in fetal alcohol syndrome and fetal alcohol effects. J Dev Behav Pediatr 25(4): 228-238. doi: 0196-206X/00/2504-0228

Streissguth, A. (2007). Offspring effects of prenatal alcohol exposure from birth to 25 years: The Seattle prospective longitudinal study. J Clin Psychol Med Settings 14(2): 81-101. doi: $10.1007 / \mathrm{s} 10880-007-9067-6$

Tamana, S., Pei, J., Massey, D., Massey, V., and Rasmussen, C. (2014). Neuropsychological Impairments and Age-Related Differences in Children and Adolescents with Fetal Alcohol Spectrum Disorders. J Popul Ther Clin Pharmacol 21(2): e167-e180.

Treit, S., Lebel, C., Baugh, L., Rasmussen, C., Andrew, G., and Beaulieu, C. (2013). Longitudinal MRI reveals altered trajectory of brain development during childhood and adolescence in fetal alcohol spectrum disorders. J. Neurosci. 33(24): 98-109. doi:

\subsection{3/JNEUROSCI.5004-12.2013}

Wechsler, D. (2003). Wechsler Intelligence Scale for Children ( $4^{\text {th }}$ ed.). San Antonio, TX: The Psychological Corporation.

Wechsler, D. (2005). Wechsler Individual Achievement Test (2 ${ }^{\text {nd }}$ ed.). San Antonio, TX: The Psychological Corporation. 
Wechsler, D. (2008). Wechsler Adult Intelligence Scale (4 ${ }^{\text {th }}$ ed.). San Antonio, TX: The Psychological Corporation.

Wechsler, D. (2009). Wechsler Individual Achievement Test ( ${ }^{\text {rd }}$ ed.). San Antonio, TX: The Psychological Corporation.

Wilkinson, G.S., and Robertson, G.J. (2006). Wide Range Achievement Test (4 ${ }^{\text {th }}$ ed.). Lutz, FL: Psychological Assessment Resources. 


\section{Figure Caption}

Figure 1. Mean comparisons examining the adolescent and school-age FASD diagnosed groups on common predictive factors.

\section{Table Legends}

Table 1. Means, Standard Deviations, and Ranges or Percentages of Demographic Information and Neuropsychological Assessment Measures for School-Age and Adolescent Diagnosed (PAEFASD) and Non-Diagnosed (PAE) Groups

Note. Stressors $=$ Psychosocial stressors and adverse life events. FSIQ $=$ Full-Scale IQ. VCI $=$ Verbal Comprehension Index. PRI $=$ Perceptual Reasoning Index. WMI $=$ Working Memory Index. PSI $=$ Processing Speed Index. $\mathrm{RCn}=$ Reading Comprehension. WR $=$ Word Reading. PD $=$ Pseudoword Decoding. $\mathrm{SP}=$ Spelling. $\mathrm{MPS}=$ Math Problem-Solving. $\mathrm{MC}=$ Math Computations. SP:T = School Problems: Teacher. ATT:P = Inattention: Parent. ATT:T = Inattention: Teacher. HYP:P = Hyperactivity/Impulsivity: Parent. HYP:T = Hyperactivity/Impulsivity: Teacher. GEC:P = Global Executive Composite: Parent. GEC:T= Global Executive Composite: Teacher. LF:TC = Letter Fluency: Total Correct. CS:TC = Category Switching: Total Correct. CS:TA = Category Switching: Total Accuracy. VS = Visual Scanning. NS $=$ Number Sequencing. LS $=$ Letter Sequencing. LNS $=$ Letter-Number Sequencing. $\mathrm{TACH}=$ Total Achievement Score. AS:P = Adaptive Skills: Parent. AS:T = Adaptive Skills: Teacher.

Table 2. Logistic Regressions Predicting the Likelihood of Receiving an FASD Diagnosis:

Neuropsychological Assessment Measures, Final Model

${ }^{a}$ Improvement in the correct classification when compared to the initial model. 
Age-Related Differences 24

Note. S.E. $=$ Standard Error. $\mathrm{R}^{2}$ Range $=$ Cox $\&$ Snell $\mathrm{R}^{2}$ to Nagelkerke R ${ }^{2}$. C.I. $(95 \%)=95 \%$ Confidence Interval. Stressors $=$ Psychosocial stressors and adverse life events. 
Table 1. Means, Standard Deviations, and Ranges or Percentages of Demographic Information and Neuropsychological Assessment Measures for School-Age and Adolescent Diagnosed (PAE-FASD) and Non-Diagnosed (PAE) Groups

\begin{tabular}{|c|c|c|c|c|c|c|c|c|c|c|c|c|}
\hline \multirow[t]{3}{*}{ DOMAIN } & \multicolumn{6}{|c|}{ SCHOOL-AGE } & \multicolumn{5}{|c|}{ ADOLESCENT } & \multirow[b]{3}{*}{$\begin{array}{c}\text { Range / } \\
\text { \% }\end{array}$} \\
\hline & \multirow[b]{2}{*}{$\mathbf{n}$} & \multicolumn{2}{|c|}{ PAE-FASD } & \multicolumn{3}{|c|}{ PAE } & \multicolumn{3}{|c|}{ PAE-FASD } & \multicolumn{2}{|r|}{ PAE } & \\
\hline & & $\begin{array}{l}\text { Mean } \\
\text { (SD) }\end{array}$ & $\begin{array}{c}\text { Range / } \\
\%\end{array}$ & $\mathbf{n}$ & $\begin{array}{l}\text { Mean } \\
\text { (SD) }\end{array}$ & $\begin{array}{c}\text { Range / } \\
\%\end{array}$ & $\mathbf{n}$ & $\begin{array}{l}\text { Mean } \\
\text { (SD) }\end{array}$ & $\begin{array}{c}\text { Range / } \\
\%\end{array}$ & $\mathbf{n}$ & $\begin{array}{l}\text { Mean } \\
\text { (SD) }\end{array}$ & \\
\hline \multicolumn{13}{|c|}{ DEMOGRAPHIC INFORMATION } \\
\hline Age (years) & 95 & $\begin{array}{r}8.48 \\
(1.64)\end{array}$ & $6.00-11.92$ & 79 & $\begin{array}{l}8.77 \\
(1.59)\end{array}$ & $6.17-11.75$ & 38 & $\begin{array}{l}14.45 \\
(1.91)\end{array}$ & $12.00-17.83$ & 26 & $\begin{array}{l}14.80 \\
(1.89)\end{array}$ & $12.00-17.92$ \\
\hline FASD Diagnosis: & 95 & & $54.6 \%$ & 79 & & $45.4 \%$ & 38 & & $59.4 \%$ & 26 & & $40.6 \%$ \\
\hline FAS & 0 & & -- & & & & 0 & & -- & & & \\
\hline pFAS & 10 & & $10.5 \%$ & & & & 2 & & $5.3 \%$ & & & \\
\hline ARND & 85 & & $89.5 \%$ & & & & 36 & & $94.7 \%$ & & & \\
\hline Sex & 95 & & & 79 & & & 38 & & & 26 & & \\
\hline Boys & 66 & & $69.5 \%$ & 44 & & $55.7 \%$ & 19 & & $50.0 \%$ & 15 & & $57.7 \%$ \\
\hline Girls & 29 & & $30.5 \%$ & 35 & & $44.3 \%$ & 19 & & $50.0 \%$ & 11 & & $42.3 \%$ \\
\hline Other Diagnoses & 74 & & $77.9 \%$ & 61 & & $77.2 \%$ & 26 & & $68.4 \%$ & 15 & & $57.7 \%$ \\
\hline
\end{tabular}




\begin{tabular}{|c|c|c|c|c|c|c|c|c|c|c|c|c|}
\hline Stressors & 94 & $\begin{array}{c}3.19 \\
(2.75)\end{array}$ & $0-11$ & 79 & $\begin{array}{l}4.39 \\
(3.13)\end{array}$ & $0-11$ & 35 & $\begin{array}{c}3.37 \\
(2.90)\end{array}$ & $0-11$ & 26 & $\begin{array}{c}4.58 \\
(3.06)\end{array}$ & $0-11$ \\
\hline Caregiver: & 95 & & & 79 & & & 38 & & & 26 & & \\
\hline Foster care & 67 & & $70.5 \%$ & 47 & & $59.5 \%$ & 19 & & $50.0 \%$ & 13 & & $50.0 \%$ \\
\hline Birth family & 10 & & $10.5 \%$ & 10 & & $12.7 \%$ & 7 & & $18.4 \%$ & 8 & & $30.8 \%$ \\
\hline Adoptive family & 9 & & $9.5 \%$ & 9 & & $11.4 \%$ & 6 & & $15.8 \%$ & 2 & & $7.7 \%$ \\
\hline Extended family & 8 & & $8.4 \%$ & 10 & & $12.7 \%$ & 4 & & $10.5 \%$ & 3 & & $11.5 \%$ \\
\hline Group home & 1 & & $1.1 \%$ & 2 & & $2.5 \%$ & 2 & & $5.3 \%$ & 0 & & -- \\
\hline Other & 0 & & -- & 1 & & $1.3 \%$ & 0 & & -- & 0 & & -- \\
\hline \multicolumn{13}{|l|}{ COGNITIVE } \\
\hline FSIQ & 90 & $\begin{array}{c}78.14 \\
(12.52)\end{array}$ & 41-108 & 74 & $\begin{array}{r}87.84 \\
(10.06)\end{array}$ & $68-116$ & 35 & $\begin{array}{c}72.83 \\
(11.16)\end{array}$ & $54-100$ & 24 & $\begin{array}{r}82.08 \\
(10.35)\end{array}$ & $60-100$ \\
\hline VCI & 93 & $\begin{array}{c}77.09 \\
(12.52)\end{array}$ & $47-104$ & 75 & $\begin{array}{l}85.93 \\
(9.80)\end{array}$ & $65-116$ & 35 & $\begin{array}{c}68.49 \\
(13.02)\end{array}$ & $47-98$ & 22 & $\begin{array}{c}82.55 \\
(12.54)\end{array}$ & $60-104$ \\
\hline PRI & 93 & $\begin{array}{r}89.54 \\
(13.89)\end{array}$ & $46-122$ & 74 & $\begin{array}{c}97.01 \\
(13.07)\end{array}$ & $70-132$ & 35 & $\begin{array}{r}83.37 \\
(14.71)\end{array}$ & $58-128$ & 22 & $\begin{array}{c}92.73 \\
(12.27)\end{array}$ & $74-111$ \\
\hline WMI & 91 & 76.84 & $54-117$ & 75 & 87.73 & $52-117$ & 35 & 78.00 & $52-114$ & 22 & 81.23 & $62-103$ \\
\hline
\end{tabular}




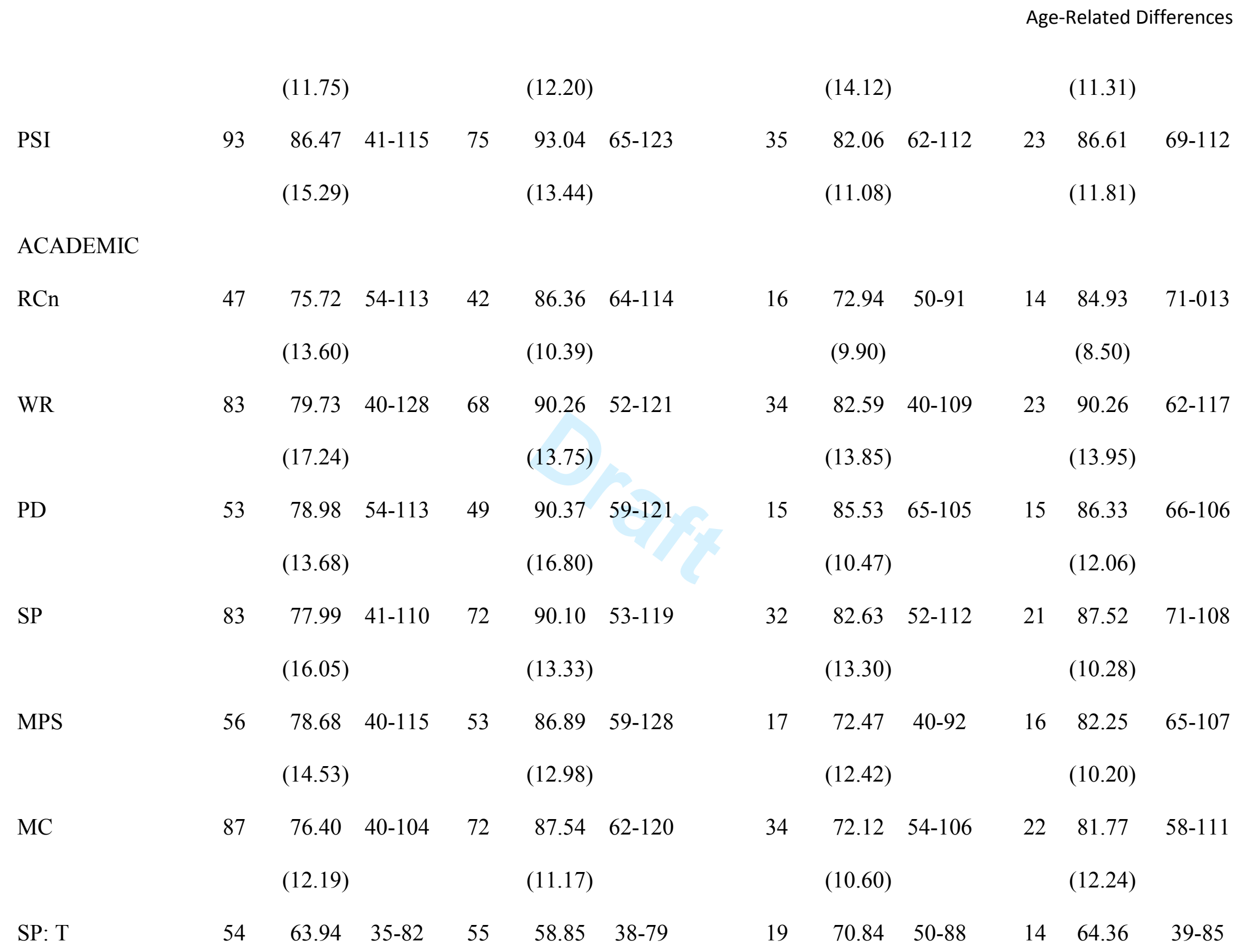


Age-Related Differences 4
$(10.22)$
(9.32)
(9.87)
$(14.62)$

ATTENTION

ATT: P

$\begin{array}{llllll}81 & 77.91 & 42-90 & 72 & 77.22 & 45-90 \\ & (13.05) & & & \\ & & & & & \end{array}$

$\begin{array}{lrrrrr}36 & 75.50 & 47-90 & 21 & 72.90 & 50-90 \\ & (12.73) & & & (13.18)\end{array}$

ATT: T

$\begin{array}{llllll}59 & 69.14 & 41-86 & 60 & 66.60 & 39-90\end{array}$

$21 \quad 78.81 \quad 52-90$

$15 \quad 71.20 \quad 41-90$

(9.45)

$(13.11)$

$(11.20)$

$(15.28)$

HYP: P

$\begin{array}{llllll}81 & 78.27 & 39-90 & 73 & 77.97 & 41-90 \\ & (14.06) & & & (13.17)\end{array}$

$36 \quad 72.56-51-90$

$\begin{array}{lll}21 & 72.57 \quad 48-90\end{array}$

$(13.52)$

$(12.81)$

HYP: T

\begin{tabular}{llllll}
59 & 69.37 & $41-90$ & 60 & 68.63 & $41-90$ \\
$(14.49)$ & & \multicolumn{3}{c}{$(16.34)$}
\end{tabular}
$21 \quad 62.57 \quad 42-90$
$(16.40)$

$15 \quad 61.27$

$44-90$

$(16.87)$

\section{EXECUTIVE FUNCTIONING}

GEC: P

\begin{tabular}{lrrrrr}
82 & 72.37 & $44-96$ & 71 & 71.49 & $38-94$ \\
& $(11.09)$ & & \multicolumn{3}{c}{$(10.44)$}
\end{tabular}

GEC: T

$$
\begin{array}{llllll}
60 & 72.12 & 42-114 & 58 & 71.84 & 43-123 \\
& (13.96) & & & & \\
& & &
\end{array}
$$
$36 \quad 74.39 \quad 50-91$
(10.24)

$22 \quad 75.05 \quad 51-94$
$\begin{array}{llllll}20 & 86.05 & 47-119 & 15 & 74.87 & 43-113\end{array}$
(18.71)
(19.62)

$\underline{\text { Verbal Fluency }}$

LF: TC

$\begin{array}{llllll}32 & 7.63 & 3-14 & 38 & 9.45 & 5-14\end{array}$

$32 \quad 6.88 \quad 2-13$

$20 \quad 7.60$

$3-11$ 
Age-Related Differences 5

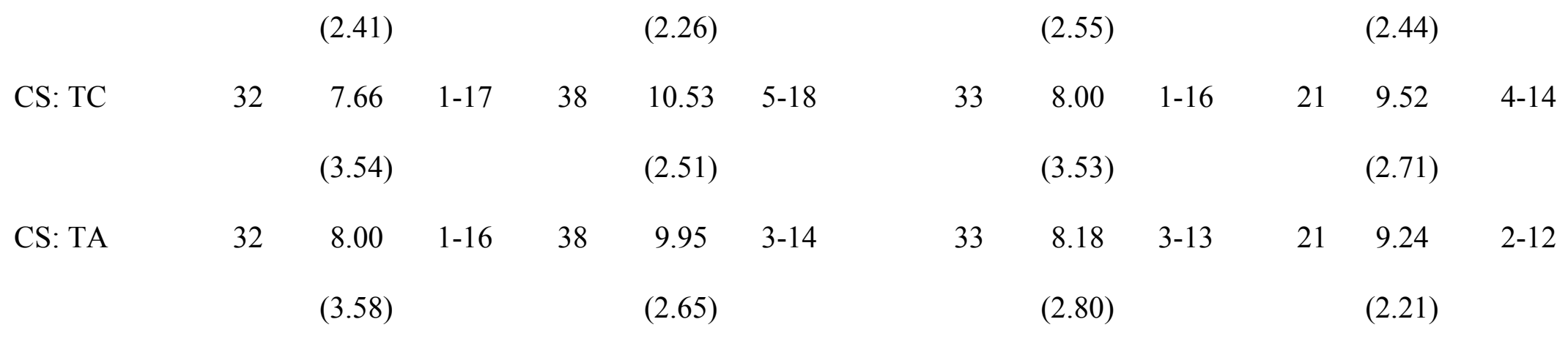

Trail Making

VS

$\begin{array}{llllll}31 & 9.94 & 1-14 & 38 & 10.16 & 2-15 \\ & (2.87) & & & (3.41) & \end{array}$

32

$8.00 \quad 3-13$

$18 \quad 10.33 \quad 5-13$

$(2.85)$

(2.11)

NS

$\begin{array}{llllll}35 & 9.46 & 1-15 & 40 & 11.58 & 5-15\end{array}$

34

9.24

4-14

$22 \quad 10.18$

6-13

(3.53)

$(2.49)$

$(2.46)$

(1.92)

LS

$\begin{array}{ll}35 & 7.77\end{array}$

1-1

$9.05 \quad 1-15$

34

$7.41 \quad 1-12$

$22 \quad 10.18$

6-13

(4.02)

(4.06)

(3.64)

(1.84)

LNS

$\begin{array}{llllll}31 & 7.72 & 1-16 & 40 & 7.60 & 2-14\end{array}$

33

$6.12 \quad 1-11$

$22 \quad 7.64$

$1-11$

(4.13)

(3.26)

$(3.01)$

(3.19)

TE LNS

$\begin{array}{llllll}31 & 8.00 & 1-12 & 39 & 8.05 & 1-12 \\ & (3.50) & & & (3.19) & \end{array}$

33

$10.03 \quad 4-12$

$22 \quad 10.23$

4-16

(2.20)

(2.45)

$\underline{\text { Tower Test }}$ 

25

$\begin{array}{lllll}9.68 & 7-13 & 34 & 10.59 & 7-14 \\ (1.93) & & & (1.73) & \end{array}$

\section{ADAPTIVE FUNCTIONING}

AS: P

72

33.19

$15-50$

66

$35.33 \quad 17-61$

34

31.4

17-49

23

32.04

$19-54$

AS: T

$52 \quad 37.04$

$25-52$

54

$42.06 \quad 27-59$

19

$35.16 \quad 23-49$

$13 \quad 43.15$

29-66

Note. Stressors $=$ Psychosocial stressors and adverse life events. FSIQ = Full-Scale IQ. VCI = Verbal Comprehension Index. PRI = Perceptual Reasoning Index. WMI $=$ Working Memory Index. PSI $=$ Processing Speed Index. $\mathrm{RCn}=$ Reading Comprehension. $\mathrm{WR}=$ Word Reading. $\mathrm{PD}=$ Pseudoword Decoding. $\mathrm{SP}=$ Spelling. MPS $=$ Math Problem-Solving. $\mathrm{MC}=$ Math Computations. SP:T $=$ School Problems: Teacher. ATT:P = Inattention: Parent. ATT:T = Inattention: Teacher. HYP:P = Hyperactivity/Impulsivity: Parent. HYP:T = Hyperactivity/Impulsivity: Teacher. GEC:P = Global Executive Composite: Parent. GEC:T = Global Executive Composite: Teacher. LF:TC = Letter Fluency: Total Correct. CS:TC = Category Switching: Total Correct. CS:TA = Category Switching: Total Accuracy. VS $=$ Visual Scanning. NS $=$ Number Sequencing. LS $=$ Letter Sequencing. LNS $=$ Letter-Number Sequencing. TACH $=$ Total Achievement Score. AS:P = Adaptive Skills: Parent. AS:T = Adaptive Skills: Teacher. 
Table 2. Logistic Regressions Predicting the Likelihood of Receiving an FASD Diagnosis: Neuropsychological Assessment Measures,

Final Model

\section{GROUP}

DOMAIN

\begin{tabular}{|c|c|c|c|}
\hline Final Model & $\mathbf{R}^{2}$ Range & Correct & Change in Correct \\
\hline
\end{tabular}

Predictor Variables

B (S.E.) Odds Ratio C.I. (95\%)

\section{SCHOOL-AGE}

COGNITIVE

$\underline{\text { Final Model }}$

$21.1 \%-28.2 \%$

$66.9 \%$

$+12.3 \%$

1. Stressors

2. Full-Scale IQ

$\underline{\text { Final Model }}$

$16.9 \%-22.6 \%$

$66.1 \%$

$+10.9 \%$

1. Stressors

2. Verbal Comprehension Index

Perceptual Reasoning Index

$\begin{array}{lll}-0.52(.19)^{* *} & 0.60 & 0.41-0.87 \\ -1.04(.23)^{* *} & 0.35 & 0.23-0.55\end{array}$

$\begin{array}{lll}-0.41(.18)^{*} & 0.67 & 0.47-0.94 \\ -0.67(.22)^{* *} & 0.51 & 0.33-0.79 \\ -0.29(.21) & 0.75 & 0.50-1.13\end{array}$

ACADEMIC ACHIEVEMENT 
Age-Related Differences 8

$\underline{\text { Final Model }}$

1. Stressors

2. Word Reading

Math Computations

$\underline{\text { Final Model }}$

$12.7 \%-17.0 \%$

1. Stressors

2. School Problems: Teacher

\section{EXECUTIVE FUNCTIONING}

$\underline{\text { Final Model }}$

$33.8 \%-45.4 \%$

1. Stressors

2. Trail Making Condition 2

Letter Fluency

Category Switching Correct

Category Switching Accuracy

ADAPTIVE FUNCTIONING

$\underline{\text { Final Model }}$

$15.8 \%-21.1 \%$

1. Stressors
$69.8 \%$
$+15.4 \%$

$\begin{array}{ccc}-0.50(.20)^{*} & 0.61 & 0.41-0.90 \\ -0.34(.22) & 0.71 & 0.46-1.10 \\ -1.02(.25)^{* *} & 0.36 & 0.22-0.59\end{array}$

$61.5 \% \quad+11.0 \%$

$\begin{array}{lll}-0.55(.21)^{* *} & 0.58 & 0.38-0.87 \\ 0.63(.22)^{* *} & 1.88 & 1.21-2.91\end{array}$

$76.1 \%+19.4 \%$

$\begin{array}{lll}-0.26(.32) & 0.77 & 0.41-1.44 \\ -0.69(.35)^{\mathrm{t}} & 0.50 & 0.25-1.01 \\ -0.56(.34) & 0.57 & 0.29-1.13 \\ -1.21(.50)^{*} & 0.30 & 0.11-0.80 \\ -0.01(.47) & 0.99 & 0.39-2.51\end{array}$

$62.3 \%+11.4 \%$ 
Age-Related Differences 9

2. Adaptive Skills: Teacher

$-0.77(.24)^{* *}$

0.46

$0.29-0.74$

\section{$\underline{\text { ADOLESCENT }}$}

\section{COGNITIVE}

$\underline{\text { Final Model }}$

$17.7 \%-23.8 \%$

$71.4 \%$

$+14.3 \%$

1. Stressors

2. Full-Scale IQ

$\begin{array}{ccc}-0.34(.31) & 0.71 & 0.39-1.30 \\ -0.95(.34)^{* *} & 0.39 & 0.20-0.76\end{array}$

$\underline{\text { Final Model }}$

$24.6 \%-33.2 \%$

$+11.1 \%$

1. Stressors

2. Verbal Comprehension Index

Perceptual Reasoning Index

ACADEMIC ACHIEVEMENT

$\underline{\text { Final Model }}$

$15.6 \%-21.1 \%$

$69.1 \%$

$+9.1 \%$

1. Stressors

2. Word Reading

Math Computations

\section{EXECUTIVE FUNCTIONING}

Final Model

$31.0 \%-42.1 \% \quad 80.9 \%$

$\begin{array}{lll}-0.28(.33) & 0.76 & 0.40-1.45 \\ -0.17(.38) & 0.84 & 0.40-1.79 \\ -0.80(.41)^{\mathrm{t}} & 0.45 & 0.20-1.01\end{array}$

$\begin{array}{ccc}-0.41(.34) & 0.67 & 0.34-1.30 \\ -1.17(.43)^{* *} & 0.31 & 0.13-0.73 \\ -0.03(.39) & 0.97 & 0.45-2.10\end{array}$


Age-Related Differences 10

1. Stressors

2. Trail Making Condition 1

Trial Making Condition 3

$\begin{array}{lll}-0.72(.39)^{\mathrm{t}} & 0.49 & 0.23-1.04 \\ -0.86(.44)^{\mathrm{t}} & 0.43 & 0.18-1.01 \\ -0.91(.53)^{\mathrm{t}} & 0.40 & 0.14-1.13\end{array}$

ADAPTIVE FUNCTIONING

Final Model $\quad 17.0 \%-23.0 \% \quad 71.9 \% \quad+12.5 \%$

1. Stressors

$-0.29(.38)$

0.45

$0.36-1.57$

2. Adaptive Skills: Teacher

1.49

$0.16-1.04$

${ }^{\mathrm{t}} p<.10,{ }^{*} p<.05, * * p<.01$

${ }^{a}$ Improvement in the correct classification when compared to the initial model.

Note. S.E. $=$ Standard Error. $\mathrm{R}^{2}$ Range $=$ Cox $\&$ Snell $\mathrm{R}^{2}$ to Nagelkerke $\mathrm{R}^{2}$. C.I. $(95 \%)=95 \%$ Confidence Interval. Stressors $=$ Psychosocial stressors and adverse life events. 


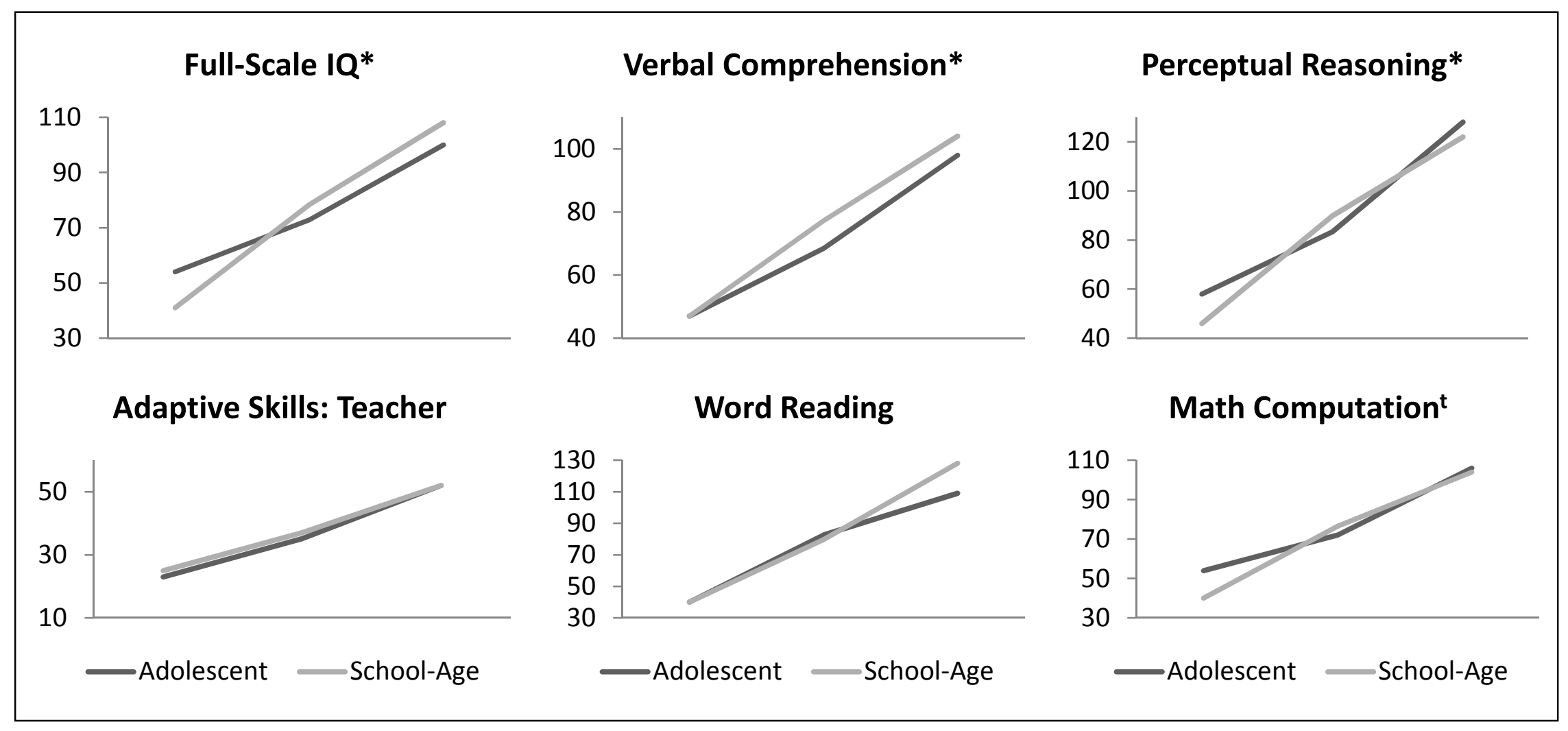

${ }^{*} p<.05,{ }^{\mathrm{t}} p<.10$

Figure 1. Mean comparisons examining the adolescent and school-age FASD diagnosed groups on common predictive factors. 